\title{
Henry Darcy et les écoulements de fluides en milieu poreux
}

\author{
C.M. Marle ${ }^{1}$ \\ 1 Université Pierre et Marie Curie, 4, place Jussieu, 75252 Paris cedex 05, France \\ e-mail : marle@math.jussieu.fr
}

\begin{abstract}
Résumé - À l'occasion du $150^{\mathrm{e}}$ anniversaire de la parution de l'ouvrage de Henry Darcy intitulé «Les fontaines publiques de la ville de Dijon» et dans lequel il énonce les principes de la loi qui porte son nom, le présent article met en perspective le rôle essentiel de la découverte de Henry Darcy dans les études d'écoulement des fluides en milieux poreux. Nous reproduisons, à la fin de cet article, avec l'aimable collaboration du service documentaire de l'École Nationale des Ponts et Chaussées, la section de l'ouvrage de H. Darcy qui décrit l'expérience soutenant l'énoncé de sa célèbre loi.
\end{abstract}

\begin{abstract}
Henry Darcy and Fluid Flows in Porous Media - To mark the 150th anniversary of the publication of the work of Henry Darcy "Les fontaines publiques de la ville de Dijon"' in which he announces the principles of the law which bears his name, this article gives a perspective on the important role played by Henry Darcy's discovery in research on fluid flow in porous media. A section extracted from H. Darcy's book, in which he describes the experiment at the origin of his famous law, is reproduced at the end of this article from a copy kindly provided by the documentation service of l'École Nationale des Ponts et Chaussées.
\end{abstract}

\section{VIE ET CEUVRE D'HENRY DARCY}

Henry Philibert Gaspard Darcy naquit le 10 juin 1803 à Dijon. Son père, fonctionnaire, mourut en 1817, alors que Henry avait 14 ans. Sa mère, Agathe Angélique Servet, réussit à faire faire de bonnes études à Henry et à son jeune frère Hugues (qui plus tard devint préfet). Henry entra à l'École Polytechnique en 1821, à l'âge de 18 ans, et choisit, à sa sortie, le Corps Impérial des Ponts et Chaussées (qui existe toujours, mais n'est bien sûr plus impérial). De 1823 à 1825 il suivit les cours de l'École des Ponts et Chaussées (créée en 1716, aujourd'hui la plus ancienne Grande École de France). Il commença sa carrière d'ingénieur en 1826, dans le Jura, puis fut affecté à Dijon. Cette ville n'avait alors pas de réseau de distribution d'eau satisfaisant. La salubrité publique en fut affectée de manière particulièrement dramatique lors de l'épidémie de choléra de 1832. En 1828, Henry Darcy épousa Henriette Carey.
Henry Darcy entreprit l'étude du captage et de la distribution de l'eau de la source du Rosoir, située à 12 kilomètres de Dijon. Remis en 1834, son projet fut accepté par le conseil municipal l'année suivante, déclaré d'utilité publique par une ordonnance royale en 1837 et, en 1838, Henry Darcy fut officiellement chargé de sa réalisation. En 1840, l'eau du Rosoir arrivait à Dijon, où elle était stockée dans deux réservoirs. Peu après, des canalisations étaient posés dans toutes les rues, et des bornes fontaines publiques étaient installées tous les 100 mètres $^{(1)}$. En 1847, l'eau était distribuée à tous les étages des bâtiments.

Henry Darcy joua un rôle décisif lors du choix du tracé de la ligne de chemin de fer Paris-Lyon-Marseille, dont la construction avait été décidée en 1842. Le tracé qu'il proposa, passant par Dijon, nécessitait le percement d'un

(1) Le cheminement administratif du projet avait duré quatre ans, et l'essentiel de sa réalisation moins de deux ans. 
tunnel de 4100 mètres, mais faisait gagner 42 kilomètres. La commission d'experts accepta ce tracé, et Henry Darcy supervisa le percement du tunnel. Le passage du chemin de fer contribua fortement au développement économique et démographique de Dijon.

Henry Darcy dut quitter Dijon lors de la révolution de 1848 , et travailla quelque temps au projet du canal du Berry, à Bourges. En 1849, il fut nommé Directeur et Ingénieur en chef du service des Eaux et des Pavés de Paris. Il fut consulté pour la création d'un réseau de distribution d'eau à Bruxelles et effectua diverses missions, notamment à Londres où il étudia le pavage des rues à base de macadam.

De retour à Dijon en 1855, Henry Darcy se consacra à des recherches expérimentales sur l'écoulement de l'eau à travers des massifs de sable, dans des conduites et dans des canaux avec surface libre. Il publia en 1856 son célèbre livre «Les fontaines publiques de la ville de Dijon » [1] (dont l'appendice D contient l'énoncé de la loi qui aujourd'hui porte son nom) et, en 1857, «Recherches expérimentales relatives au mouvement de l'eau dans les tuyaux » (où apparaît, pour la première fois, le concept de couche limite, aujourd'hui d'une grande importance en Mécanique des fluides).

Élu membre de l'Académie des Sciences en 1857, il succéda au mathématicien Augustin-Louis Cauchy dans la fonction de Président de cette prestigieuse Société savante. Il mourut à Paris d'une pneumonie le 2 janvier 1858. Son corps fut ramené à Dijon et reçut des funérailles nationales.

Pour plus de détails sur la vie de Henry Darcy, le lecteur pourra consulter [2-5].

\section{LES ÉCOULEMENTS MONOPHASIQUES EN MILIEU POREUX}

La loi de Darcy, formulée pour la première fois dans l'appendice D de [1], reste de nos jours un élément essentiel de la description mathématique de l'écoulement d'un fluide dans un milieu poreux. Elle est toujours largement utilisée dans de nombreux domaines : hydrologie, génie chimique, exploitation des gisements d'hydrocarbures... Henry Darcy l'a formulée pour l'écoulement d'eau à travers un cylindre rempli de sable, d'axe vertical, sous la forme suivante :

$$
Q=K S \frac{\Delta h}{e},
$$

où $Q$ est le débit, $S$ l'aire de la section droite et $e$ la hauteur du cylindre rempli de sable, $\Delta h$ la différence de charge hydraulique entre les extrémités inférieure et supérieure du cylindre. Le coefficient $K$ dépend de propriétés du massif de sable, telles que sa granulométrie. Il s'agit donc d'une loi linéaire, exprimant la proportionnalité entre le débit par unité de section $Q / S$ et le quotient de la différence de charge par la hauteur $\Delta h / e$. Plusieurs lois de même type, exprimant la proportionnalité entre un flux et le déséquilibre qui cause ce flux, étaient déjà connues en 1856 : loi de Fourier pour la conduction de la chaleur (1807), loi d'Ohm pour la conduction de l'électricité (1827), loi de Poiseuille pour l'écoulement laminaire d'un fluide dans un tube cylindrique (1844), loi de Fick pour la diffusion (1855). Très probablement, Henry Darcy connaissait certaines de ces lois et s'en est inspiré; peut-être même a-t-il été élève de Jean-Baptiste Joseph Fourier (1768-1830), qui fut Professeur à l'École Polytechnique et publia son traité « Théorie analytique de la chaleur » en 1816.

Initialement globale (valable pour un massif poreux homogène et un écoulement vertical uniforme), la formulation de la loi de Darcy devint rapidement locale, applicable à des corps poreux hétérogènes, à des fluides autres que l'eau, éventuellement compressibles, et à des écoulements non uniformes. Aujourd'hui, elle a la forme suivante :

$$
\vec{u}=-\frac{k}{\mu}(\overrightarrow{\operatorname{grad}} p-\rho \vec{g}),
$$

où $\vec{u}$ est la vitesse de filtration (vecteur flux volumique de fluide), $\overrightarrow{\operatorname{grad}} p$ le vecteur gradient de la pression $p, \rho$ la masse volumique du fluide, $\mu$ sa viscosité, $\vec{g}$ le vecteur accélération de la pesanteur et $k$ un coefficient, homogène à une surface, pouvant avoir un caractère tensoriel, dépendant uniquement du milieu poreux, appelé perméabilité. L'unité pratique de perméabilité, toujours largement utilisée bien qu'elle ne fasse pas partie d'un système d'unités cohérent, est le darcy. Il importe d'observer que les grandeurs qui figurent dans cette équation peuvent dépendre du point de l'espace considéré, voire aussi du temps. Ce sont des grandeurs locales, mais macroscopiques, ayant un sens non à l'échelle des pores, mais à l'échelle d'un bloc élémentaire de milieu poreux.

Sous cette forme généralisée, la loi de Darcy est très bien vérifiée par l'expérience, du moins dans un certain domaine : les déformations du milieu poreux doivent être négligeables, et l'écoulement du fluide, à l'échelle des pores, doit être bien décrit par les équations de Navier-Stokes (équations de Navier linéarisées dans lesquelles les termes représentant des forces d'inertie sont négligés, ce qui suppose l'écoulement suffisamment lent). La validité des équations de Navier-Stokes suppose aussi le fluide homogène, de composition constante, le libre parcours moyen des molécules du fluide petit auprès de la dimension des pores, et les phénomènes d'adsorption du fluide sur le solide négligeables. Pour une étude critique approfondie des conditions de validité de la loi de Darcy, le lecteur pourra consulter le livre d'André Houpeurt [6]. Lorsque toutes ces conditions ne sont pas satisfaites, on peut encore souvent utiliser la loi de Darcy en lui ajoutant des termes correctifs.

Outre la loi de Darcy, les grandeurs qui décrivent, à l'échelle macroscopique, les propriétés de l'écoulement doivent aussi vérifier une relation exprimant la conservation de la masse du fluide, appelée équation de continuité, qui 
s'écrit (pour un milieu poreux parfaitement rigide et immobile et un fluide isotherme de composition constante)

$$
\operatorname{div}(\rho \vec{u})+\Phi \frac{\partial \rho}{\partial t}=0
$$

où div est l'opérateur divergence (appliqué au flux massique de fluide, $\vec{\rho} \vec{u}$ ), $\Phi$ la porosité du milieu (proportion, en volume, de l'espace occupé par le fluide, supposée invariable au cours du temps) et $t$ le temps.

En hydrologie et dans l'exploitation des gisements d'hydrocarbures, on peut souvent considérer le fluide comme faiblement compressible, lorsque la pression varie peu, en restant voisine d'une pression de référence $p_{0}$. La masse volumique $\rho$ est alors liée à la pression par une relation de la forme

$$
\rho=\rho_{0} \exp \left(\lambda\left(p-p_{0}\right)\right)
$$

où $\lambda$ est la compressibilité du fluide. On peut alors former, à partir de cette relation, de l'équation de Darcy et de l'équation de continuité, une équation aux dérivées partielles ne contenant plus qu'une seule fonction inconnue, la pression. Dans un milieu homogène, et lorsqu'on néglige les termes non linéaires, cette équation est de la forme

$$
\Delta p-2 \rho_{0} \lambda \overrightarrow{\operatorname{grad}} p \cdot \vec{g}=\frac{1}{\kappa} \frac{\partial p}{\partial t},
$$

où le coefficient $\kappa$ (parfois appelé coefficient de diffusivité) a pour expression

$$
\kappa=\frac{k}{\mu \lambda \Phi} .
$$

C'est une équation de même type que celle qui régit la diffusion ou la propagation de la chaleur (c'est même exactement l'équation de la chaleur lorsqu'on peut négliger les effets de la pesanteur). Elle fut largemment utilisée, tant en hydrologie que dans l'exploitation des gisements d'hydrocarbures, jusque vers les années 1960, car de nombreuses solutions exactes en sont connues, et conviennent pour décrire l'écoulement d'un fluide légèrement compressible dans une couche poreuse homogène. Le lecteur intéressé pourra consulter, par exemple, le très beau livre d'André Houpeurt [7], fondateur et premier directeur de la division Forage-Production de l'Institut français du pétrole. Aujourd'hui, grâce aux progrès de l'informatique, on peut résoudre numériquement les équations qui régissent les écoulements en milieu poreux sans avoir à supposer le milieu homogène, et sans négliger les termes non linéaires. Mais l'équation de Darcy est toujours utilisée!

\section{LES ÉCOUPEMENTS POLYPHASIQUES}

La loi de Darcy s'applique à l'écoulement d'une phase fluide unique, occupant tout l'espace poreux du milieu. Son extension aux écoulement de deux phases fluides qui se partagent l'espace poreux a été proposée, vers la fin des années 1930, par Leverett [8] ainsi que Wyckoff et Botset [9]. Il s'agit d'une généralisation très naturelle, que les auteurs cités ont validée expérimentalement. On a alors deux équations de Darcy, une pour chaque phase, de la forme

$$
\vec{u}_{i}=-\frac{k K_{i}}{\mu_{i}}\left(\overrightarrow{\operatorname{grad}} p_{i}-\rho_{i} \vec{g}\right)
$$

La plupart des symboles apparaissant dans ces équations ont la même signification que ceux qui figurent dans l'équation de Darcy usuelle du paragraphe précédent; l'indice $i$ (qui prend les valeurs 1 ou 2) indique la phase à laquelle ils se rapportent. Les deux nouveaux coefficients qui apparaissent, $K_{i}(i=1$ ou 2$)$ sont appelés perméabilités relatives (à la phase $i$ ). On admet généralement que ce sont des fonctions de la saturation $S_{i}$ (proportion de l'espace poreux occupée par la phase $i$ ). On remarquera que deux pressions, $p_{1}$ et $p_{2}$, figurent dans ces équations; la différence $p_{2}-p_{1}$, appelée pression capillaire, est, elle aussi, considérée comme une fonction de la saturation.

La validité de ces équations a donné lieu à de nombreuses discussions (voir par exemple [6]); elle est beaucoup plus douteuse que celle de l'équation de Darcy des écoulements monophasiques, et diverses corrections ont été proposées. Cependant, ce sont toujours ces équations qui sont utilisées pour la modélisation des écoulements dans les gisements d'hydrocarbures.

\section{CONCLUSION}

Henry Darcy fut un très grand ingénieur et un remarquable pionnier de la recherche expérimentale appliquée. Homme désintéressé, il a, pendant toute sa carrière, œuvré à l'amélioration des conditions de vie de ses concitoyens. Il a écrit son célèbre livre [1] afin de transmettre son savoirfaire, de permettre à d'autres de réaliser, dans d'autres villes, des réseaux de distribution d'eau aussi performants que celui qu'il avait créé à Dijon. Ce livre a récemment été traduit en anglais par Patricia Bobeck et réédité aux États-Unis [10]. En France, il est introuvable en librairie et ne peut être consulté que dans certaines bibliothèques spécialisées. Heureusement, la Bibliothèque Nationale, qui a entrepris un remarquable effort de numérisation et de mise en ligne gratuite de documents, l'a placé sur son site. On peut le télécharger gratuitement à l'adresse http ://gallica.bnf.fr

à condition de disposer d'une connexion internet rapide et de beaucoup de patience (il ne semble pas possible pour le moment de télécharger le livre entier en une seule fois, il faut procéder page par page).

Les hydrologues ont récemment honoré Henry Darcy à l'occasion du Colloque international [11]. Il est heureux que les pétroliers le fassent aussi, en publiant dans cette Revue un extrait de son célèbre livre. 


\section{RÉFÉRENCES}

1 H. Darcy (1856) Les fontaines publiques de la ville de Dijon; exposition et application des principes à employer dans les questions de distribuion d'eau. Ouvrage terminé par un appendice relatif aux fournitures d'eau de plusieurs villes, au filtrage des eaux et à la fabrication des tuyaux de fonte, de plomb, de tôle et de bitume. Victor Dalmont, éditeur, Paris.

2 P. Bobeck (2006) Henry Darcy's Public Fountains of the City of Dijon, Communication au Colloque international Gestion des grands aquifères [11].

3 É. Lochot (2006) Henry Darcy et les fontaines publiques de la ville de Dijon, Communication au Colloque international Gestion des grands aquifères [11].

4 P. Rat (2006) Darcy, l'eau à Dijon et l'air du temps, Communication au Colloque international Gestion des grands aquifères [11].

5 C.T. Simmons (2006) Henry Darcy (1803-1858) : Immortalized by his scientific legacy, Communication au Colloque international Gestion des grands aquifères [11].
6 A. Houpeurt (1974) Mécanique des fluides dans les milieux poreux, critique et recherche, éditions Technip, Paris.

7 A. Houpeurt (1974) Éléments de mécanique des fluides dans les milieux poreux, éditions Technip, Paris.

8 M.C. Leverett (1938) Flow of oil-water mixtures through unconsolidated sands, T. AIME 132, 149.

9 R.D. Wyckoff and H.G. Botset (1936) The flow of gas-liquid mixtures through unconsolidated sands, Physics 7, 325.

10 H. Darcy (2004) The Public Fountains of the City of Dijon. English translation by Patricia Bobeck, Kendall Hunt Pub. Co., Dubuque (Iowa).

11 Association Internationale des Hydrogéologues (2006) Gestion des grand aquifères. Colloque international, Dijon, 30 mai au 1er juin 2006. Les actes de ce Colloque peuvent être téléchargés sur le site : http ://www.brgm.fr/brgm/aih/presentation.htm

Manuscrit final reçu en septembre 2006 or distributed for profit or commercial advantage and that copies bear this notice and the full citation on the first page. Copyrights for components of this work owned by others than IFP must be honored. Abstracting with credit is permitted. To copy otherwise, to republish, to post on servers, or to redistribute to lists, requires prior specific permission and/or a fee: Request permission from Documentation, Institut français du pétrole, fax. +33147527078 , or revueogst@ifp.fr. 


\title{
FONTAINES PUBLIOUES
}

\section{DE LA VILLE DE DIJON}

\author{
EXPOSITION ET APPLICATION \\ DES PRINCIPES A SUIVRE ET DES FORMULES A EMPLOYER \\ DANS LES QUESTIONS \\ DISTRIBUTION D'EAU \\ OOVRAGB TBRHIISE

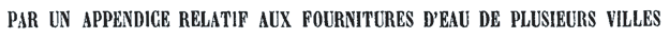 \\ AU FILTRAGE DES EAUX

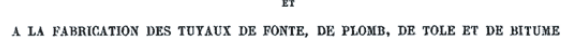 \\ PAR \\ HENRY DARCY \\ INSPECTEUR GÉNÉRAL DES PONTS ET EHAUSSÉES.

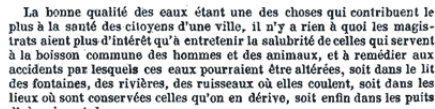 \\ des fontaines, des rivieres, des ruisseaux où elles coulent, soit dans lee

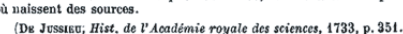 \\ PARIS \\ VICTOR DALMONT, EDITEUR, \\ Successeur de Carilian-Gicury et lor Dalmont,

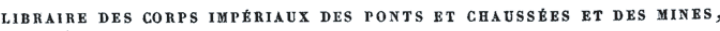 \\ Quai des Augustins, 49. \\ $\overline{1856}$
}

H. Darcy, inspecteur général des Ponts et Chaussées, présentait dans ce livre de plus de 600 pages une synthèse « des principes à employer dans les questions de distribution d'eau », pour reprendre le sous-titre. Dans cinq pages (pp. 590 à 594 ) et une figure (planche 24, figure 3) de l'appendice - note D, il exposait les résultats d'une expérience exemplaire dans sa simplicité. Il démontrait, par l'intermédiaire de « $k$ un coefficient dépendant de la perméabilité de la couche », la proportionnalité entre débit et perte de charge dans un filtre sableux. Cent cinquante ans plus tard, de très nombreux hydrauliciens ou ingénieurs pétroliers utilisent toujours le symbole $k$ pour la perméabilité dont l'unité CGS est le Darcy. Bien peu d'entre eux ont eu la possibilité de lire ces cinq pages. C'est cette possibilité que nous voudrions offrir à nos lecteurs en donnant ici leur fac-simile. Nous remercions la bibliothèque de l'École Nationale des Ponts et Chaussées de nous avoir permis de photographier ce document.

Le comité de rédaction

H. Darcy, Inspector General of Bridges and Highways, presented in this book of over 600 pages a summary of the "Principles to be used in the question of the distribution of water"; which is the sub-title of the book. In 5 pages (p. 590 to $p$. 594) and a diagram (Plate 24, Fig. 3) of the Appendix - note D, he explained the results of an experiment which is exemplary in its simplicity. He showed, through the intermediate of " $k$, a coefficient dependent on the permeability of the layer", that the flow rate is proportional to the pressure drop in a sand filter. One hundred and fifty years later, for permeability, a large number of hydraulic and petroleum engineers still use the symbol $k$ whose CGS unit is the Darcy. Only a few of these engineers have had the possibility to read these five pages. We would like to offer this possibility to our readers by proposing their facsimile. We would like to thank the library at the École Nationale des Ponts et Chaussées for giving us their kind permission to photograph this document.

The Editorial Board 
table de la rivière, si, en un mot, la perte de charge subie par les eaux, au moment de leur pénétration à travers le radier, est grande, on comprend, dans ce cas, que la diminution de hauteur procurée par l'élargissement du radier à l'eau qui le surmonte serait à peu près insignifiante en présence de la perte de charge nécessaire à l'introduction du volume primitif. Cette diminution de hauteur ne pourrait donc apporter au débit de la galerie aucune amélioration sensible.

On peut, au reste, se rendre à peu près compte de l'accroissement de débit qu'un élargissement de galerie entraînerait; il suffirait pour cela d'établir une retenue à l'extrémité aval de la galerie, et de déterminer, au moyen de l'abaissement successif du niveau de cette retenue, la loi qui lie les accroissements du volume aux diminutions de hauteur d'eau sur le radier. Cette loi, comme on le sait, sera linéaire.

Et l'on déduira, de l'abaissement probable qui résulterait de l'élargissement du radier, l'accroissement de volume que cet abaissement entraînera. Il est facile d'obtenir immédiatement le cas limite, celui où la largeur du radier étant infinie, la hauteur d'eau sur sa surface pourrait être considérée comme nulle; et si, dans cette hypothèse extrême, on n'arrive qu'à un accroissement très-faible dans le débit, on n’aura pas à songer davantage à aúgmenter la largeur de la galerie.

Profondeur des galeries. - L'approfondissement des galeries présente des chances certaines d'accroissement de produit, et ces accroissements peuvent être mesurés d'une manière très-approchée (je suppose toujours la couche à traverser homogène), par le moyen indiqué plus haut pour déterminer la loi qui lie les diminutions de hauteur d'eau sur le radier aux accroissements du volume.

Je bornerai ici ce que j'avais à dire sur les grands filtres artificiels ou naturels. Quant aux filtres de ménage et des fontaines marchandes de la ville de Paris, on pourra recourir, si on veut en connaître les dispositions, à l'ouvrage de M. Dupuit sur les distributions d'eau.

Déterminatlon des lois d'écoulement de l'eau à travers lo sable.

J'aborde maintenant le récit des expériences que j'ai faites à Dijon de concert avec M. l'ingénieur Charles Ritter, pour déterminer les lois de l'écoulement de l'eau à travers les sables. Les expériences ont été répétées par M. l'ingénieur en chef Baumgarten.

L'appareil employé pl. 24, fig. 3, consistait en une colonne verticale de $2^{\mathrm{m}} 50$ de hauteur, formée d'une portion de conduite de $0^{\mathrm{m}} 35$ de diamètre intérieur, et close à chacune de ses extrémités par une plaque boulonnée.

A l'intérieur, et à $0^{\mathrm{m}} 20$ au-dessus du fond, se trouve une cloison horizontale à 
claire-voie, destinée à supporter le sable, et qui divise la colonne en deux chambres. Cette cloison est formée par la superposition de bas en haut d'une grille en fer à barreaux prismatiques de $0^{\mathrm{m}} 007$, d'une grille à barreaux cylindriques de $0^{\mathrm{m}} 005$, enfin d'une toile métallique à mailles de $0^{\mathrm{n}} 002$. L'écartement des barreaux de chacune des grilles est égal à leur épaisseur, et les deux grilles sont disposées de façon que leurs barreaux soient dans des directions perpendiculaires l'une à l'autre.

La chambre supérieure de la colonne recoit l'eau par un tuyau embranché sur la conduite de l'hôpital, et dont un robinet permet de modérer à volonté le débit; la chambre inférieure s'ouvre par un robinet sur un bassin de jaugeage de 1 mètre de côté.

La pression aux deux extrémités de la colonne est indiquée par des manomètres à mercure en $\mathrm{U}$; enfin chacune des chambres est munie d'un robinet à air, essentiel pour la mise en charge de l'appareil.

Les expériences ont été faites avec du sable siliceux de Saône, composé ainsi qu'il suit :

$$
\begin{aligned}
& 0^{\mathrm{m}} 58 \text { de sable passant au crible de } 0^{\text {mil. }} 77 \\
& \begin{array}{lllll}
0^{\mathrm{m}} 13 & - & - & & 1
\end{array} \\
& 0^{\mathrm{m}} 12 \quad-\quad-\quad 2 \quad 200 \\
& 0^{\mathrm{m}} 17 \text { de menu gravier, débris de coquilles, etc. }
\end{aligned}
$$

Il présente environ $\frac{38}{100}$ de vide.

Le sable était versé et tassé dans la colonne préalablement remplie d'eau, afin que les vides de la masse filtrante ne continssent plus d'air, et la hauteur du sable n'était mesurée qu'à la fin de chaque série d'expériences, après que le passage de l'eau l'avait convenablement tassé.

Chaque expérience consistait à établir dans la chambre supérieure de la colonne, par la manœuvre du robinet d'amenée, une pression déterminée; puis, lorsque par deux observations l'on s'était assuré que l'écoulement était devenu sensiblement uniforme, on notait le débit du filtre pendant un certain temps et on en concluait le débit moyen par minute.

Pour de faibles charges, le repos presque complet du mercure du manomètre permettait d'apprécier le millimètre, représentant $26^{\text {mil. }} 2$ d'eau; lorsqu'on opérait sous de fortes pressions, le robinet d'amenée était presqu'entièrement ouvert; et alors le manomètre, malgré le diaphragme dont il était muni, présentait des oscillations continuelles; néanmoins, les fortes oscillations n'étaient qu'accidentelles, et on pouvait apprécier, à 5 millimètres près, la hauteur moyenne du mercure, c'est-à-dire connaître la pression en eau à $1^{\mathrm{m}} 30$ près.

Toutes ces oscillations manométriques étaient dues aux coups de bélier pro- 
duits par le jeu des nombreuses bornes-fontaines de l'hôpital, lieu où était placé l'appareil expérimental.

Toutes les pressions ont été rapportées au niveau de la face inférieure du filtre, et on n'a tenu aucun compte du frottement dans la partie supérieure de la colonne, lequel était évidemment négligeable.

Tableau des expériences faites à mijon les 29 et 30 octobre ot novembre 1858.

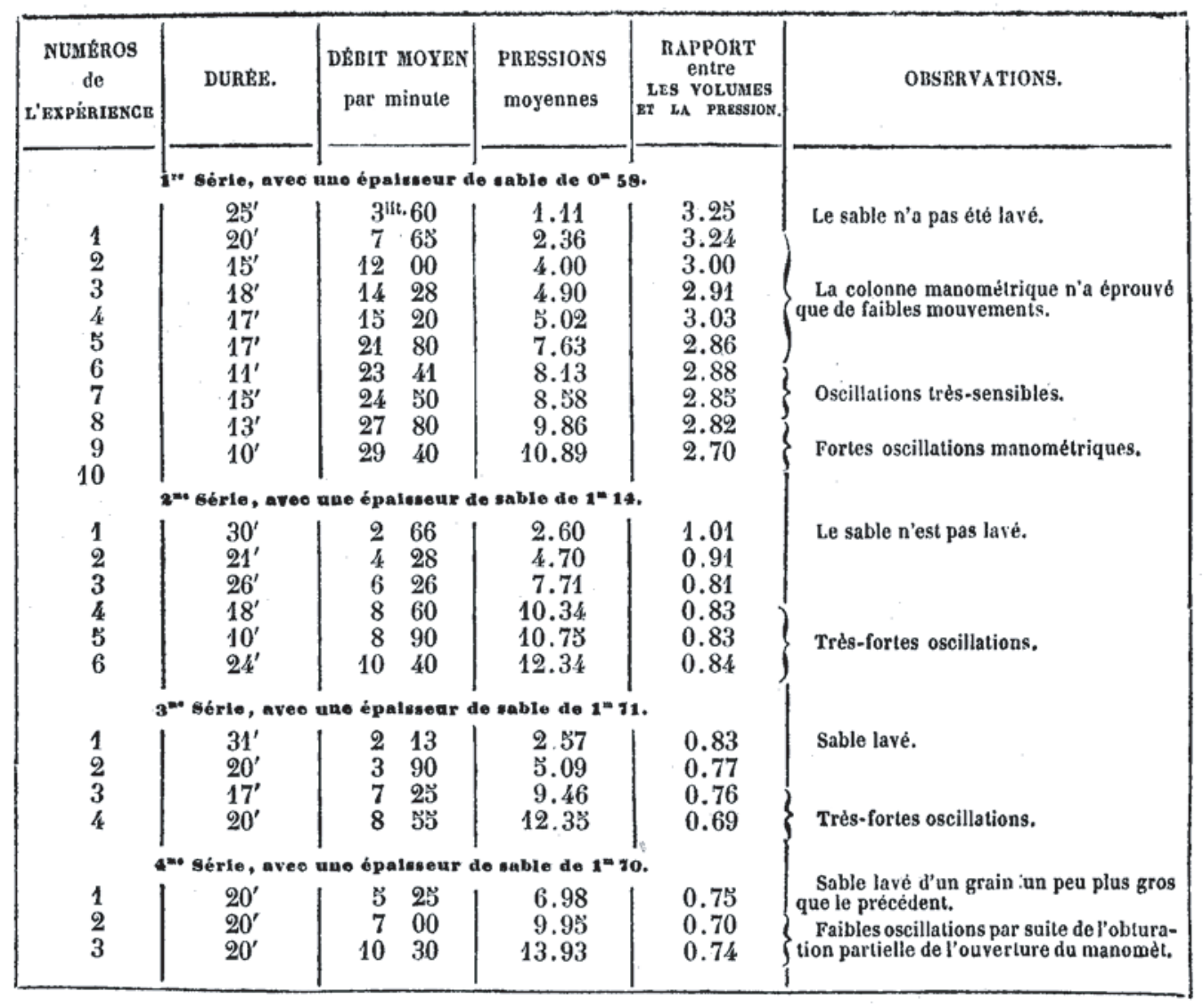

Le tableau des expériences, ainsi que leur représentation graphique, démontrent que le débit de chaque filtre crô̂t proportionnellement à la charge.

Pour les filtres sur lesquels on a opéré, le débit par seconde et par mètre carré est lié très-approximativement à la charge par les relations suivantes :

$$
\begin{array}{ll}
1^{\text {re }} \text { série. } \ldots Q=0,493 \mathrm{P} & 3^{\text {me }}-\ldots Q=0,126 \mathrm{P} \\
2^{\text {me }}-\ldots Q=0,145 \mathrm{P} & 4^{\text {me }}-\ldots Q=0,123 \mathrm{P} .
\end{array}
$$


En appelant I la charge proportionnelle par mètre d'épaisseur du filtre, ces formules se transforment dans les suivantes:

$$
\begin{array}{ll}
1^{\text {re }} \text { série. } . Q=0,286 \mathrm{I} & 3^{\mathrm{me}}-\ldots Q=0,216 \mathrm{I} \\
2^{\mathrm{me}}-\ldots Q=0,165 \mathrm{I} & 4^{\mathrm{me}}-\ldots Q=0,332 \mathrm{I} .
\end{array}
$$

Les différences entre les valeurs du coefficient $\frac{Q}{\mathrm{I}}$ proviennent de ce que le sable employé n'a pas été constamment homogène. Pour la $2^{\text {me }}$ série, il n'avait pas été lavé; pour la $3^{\text {me }}$, il était lavé; pour la $\mathbf{4}^{\text {me }}$, il était très-bien lavé et d'un grain un peu plus fort.

Il paraît donc que, pour un sable de même nature, on peut admettre que le volume débité est proportionnel à la charge et en raison inverse de l'épaisseur de la couche traversée.

Dans les expériences précédentes, la pression sous le filtre a toujours été égale à celle de l'atmosphère; il était intéressant de rechercher si la loi de proportionnalité que l'on vient de reconnaître entre les volumes débités et les charges qui les produisent subsistait encore, lorsque la pression sous le filtre était plus grande ou plus petite que la pression atmosphérique: tel est le but des expériences nouvelles opérées les 17 et 18 février 1856 par les soins de M. Ritter.

Ces expériences sont rapportées dans le tableau synoptique suivant: la colonne 4 donne les pressions sur le filtre; la colonne 5 les pressions sous le filtre, tantôt plus grandes et tantôt plus petites que le poids $\mathrm{P}$ de l'atmosphère; la colonne 6 présente les différences des pressions; enfin la colonne 7 indique

\begin{tabular}{|c|c|c|c|c|c|c|c|}
\hline $\begin{array}{c}\text { NuMÉro } \\
\text { de } \\
\text { L'axpérisscr } \\
1 \\
\end{array}$ & DURÉE. & $\begin{array}{c}\text { DÉBIT } \\
\text { M OY E N } \\
\text { par minute. } \\
\mathbf{3} \\
\end{array}$ & $\begin{array}{c}\text { PRESSION } \\
\text { SUR LE FILTRE } \\
\end{array}$ & $\begin{array}{c}\text { SOUS LE FILTRE } \\
5 \\
\end{array}$ & $\begin{array}{c}\text { DIFFÉRENCE } \\
\text { des } \\
\text { PRESSIONS. } \\
6 \\
\end{array}$ & $\begin{array}{c}\text { RAPPORT } \\
\text { des } \\
\text { volumES } \\
\text { aux } \\
\text { pressions. } \\
7 \\
7\end{array}$ & OBSERVATIONS. \\
\hline $\begin{array}{r}1 \\
2 \\
3 \\
4 \\
5 \\
6 \\
7 \\
8 \\
9 \\
10 \\
11 \\
12\end{array}$ & $\begin{array}{l}15^{\prime} \\
15^{\prime} \\
10^{\prime} \\
10^{\prime} \\
20^{\prime} \\
16^{\prime} \\
15^{\prime} \\
15^{\prime} \\
20^{\prime} \\
20^{\prime} \\
20^{\prime} \\
20^{\prime}\end{array}$ & $\begin{array}{c}1 . \\
18,8 \\
18,3 \\
18,0 \\
17,4 \\
18,1 \\
14,9 \\
12,1 \\
9,8 \\
7,9 \\
8,63 \\
4,3 \\
4,15\end{array}$ & $\begin{array}{l}\mathrm{m} . \\
\mathrm{P}+9,48 \\
\mathrm{P}+12,88 \\
\mathrm{P}+9,80 \\
\mathrm{P}+12,87 \\
\mathrm{P}+12,80 \\
\mathrm{P}+8,86 \\
\mathrm{P}+12,84 \\
\mathrm{P}+6,71 \\
\mathrm{P}+12,81 \\
\mathrm{P}+5,58 \\
\mathrm{P}+2,98 \\
\mathrm{P}+12,86\end{array}$ & $\begin{array}{l}\mathrm{p}-3,60 \\
\mathrm{p} 0 \\
\mathrm{P}-\mathbf{2}, 78 \\
\mathrm{P}+0,46 \\
\mathrm{p}+0,49 \\
\mathrm{P}-0,83 \\
\mathrm{P}+4,40 \\
\mathrm{P} \quad 0 \\
\mathrm{P}+7,03 \\
\mathrm{P} \quad 0 \\
\mathrm{P} \quad 0 \\
\mathrm{P}+9,88\end{array}$ & $\begin{array}{r}\mathrm{m} . \\
13,08 \\
12,88 \\
12,58 \\
12,41 \\
12,35 \\
9,69 \\
8,44 \\
6,71 \\
5,78 \\
5,58 \\
2,98 \\
2,98\end{array}$ & $\begin{array}{l}1,44 \\
1,42 \\
1,43 \\
1,40 \\
1,47 \\
1,54 \\
1,43 \\
1,46 \\
1,37 \\
1,55 \\
1,51 \\
1,39\end{array}$ & $\begin{array}{l}\text { Fortes oscillations dans le ma- } \\
\text { nométre supérieur. } \\
\qquad \begin{array}{l}\text { Id. } \\
\text { Id. }\end{array} \\
\text { Faibles. } \\
\text { Assez faibles. } \\
\text { Presque nulles. } \\
\text { Très-forles. } \\
\text { Très-faibles. } \\
\text { Très-fortes. } \\
\text { Presque nulles. } \\
\quad \text { Id. } \\
\text { Assez fortes. } \\
\text { On a déjà expliqué la cause de } \\
\text { ces oscillations. }\end{array}$ \\
\hline
\end{tabular}
les rapports des volumes débités aux différences des pressions existant sur et sous le filtre. L'épaisseur de la couche de sable traversée était égale à $1^{\mathrm{m}} 10$. 
La constance des rapports de la $7^{\circ}$ colonne témoigne de la vérité de la loi déjà énoncée: on remarquera cependant qu'ici encore les pressions sur et sous le filtre comprennent des limites très-étendues : sous le filtre, en effet, la pression a varié de $\mathrm{P}+9,88$ à $\mathrm{P}-3,60$, et sur le filtre de $\mathrm{P}+12,88$ à $\mathrm{P}+2,98$.

Ainsi, en appelant $e$ l'épaisseur de la couche de sable, $s$ sa superficie, P la pression atmosphérique, $h$ la hauteur de l'eau sur cette couche, on aura $\mathrm{P}+h$ pour la pression d̀ laquelle sera soumise la base supérieure; soient, de plus, $\mathrm{P} \pm h_{0}$ la pression supportée par la surface inférieure, $k$ un coefficient dépendant de la perméabilité de la couche, $q$ le volume débité, on a

$$
q=k_{e}^{s}\left[h+e \mp h_{0}\right] \text { qui se réduit à } q=k \frac{s}{e}(h+e)
$$

quand $h_{0}=0$, ou lorsque la pression sous le filtre est égale à la pression atmosphérique.

Il est facile de déterminer la loi de décroissance de la hauteur d'eau $h$ sur le filtre; en effet, soit $d h$ la quantité dont cette hauteur s'abaisse pendant un temps $d t$, sa vitesse d'abaissement sera $-\frac{d h}{d t}$; mais l'équation ci-dessus donne encore pour cette vitesse l'expression

$$
\frac{q}{s}=v=\frac{k}{e}(h+e)
$$

On aura donc $-\frac{a h}{a t}=\frac{k}{e}(h+e)$; d'où $\frac{d h}{(h+e)}=-\frac{k}{e} d t$,

et

$$
l(h+e)=\mathrm{C}-\frac{k}{e} t
$$

Si la valeur $h_{0}$ correspond au temps $t_{0}$ et $h$ d̀ un temps quelconque $t$, il viendra

$$
l(h+e)=l\left(h_{0}+e\right)-\frac{k}{e}\left[t-t_{0}\right]
$$

Si on remplace maintenant $h+e$ et $h_{0}+e \operatorname{par} \frac{q e}{s k}$ et $\frac{q_{0} e}{s k}$, il viendra

$$
l q=l q_{0}-\frac{k}{e}\left(t-t_{0}\right)
$$

et les deux équations (1) et (2) donnent, soit la loi d'abaissement de la hauteur sur le filtre, soit la loi de variation des volumes débités à partir du temps $t_{0}$.

Si $k$ et $e$ étaient inconnus, on voit qu'il faudrait deux expériences préliminaires pour faire disparaître de la seconde le rapport inconnu $\frac{k}{e}$. 
Fig. 3.

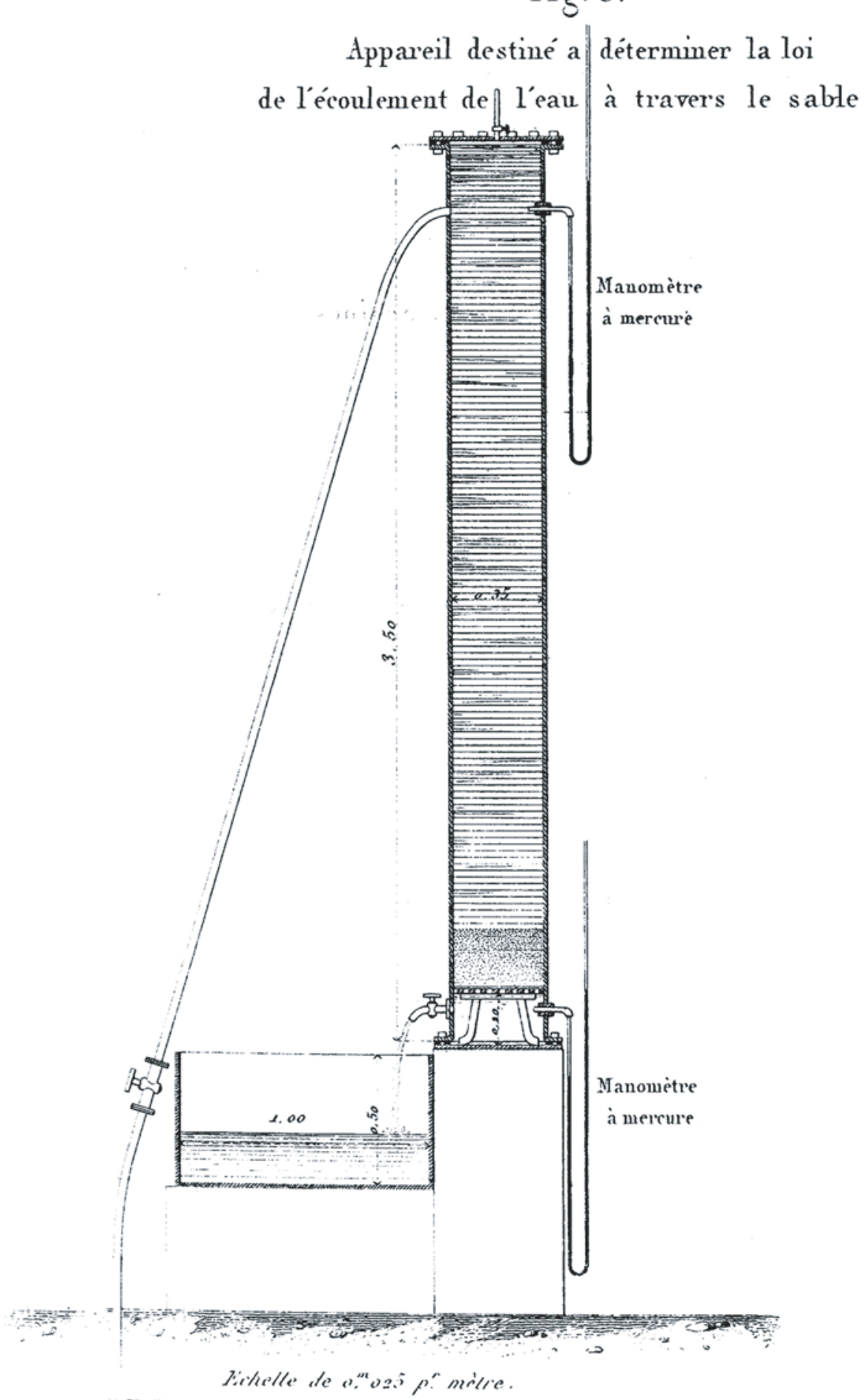

